Pirineos. Revista de Ecología de Montaña

Vol. 175

Jaca, Enero-Diciembre, 2020, e060

ISSN-1: 0373-2568

https://doi.org/10.3989/pirineos.2020.175010

\title{
SABERES LOCALES EN HUERTAS DE MONTAÑA DEL SUR DE LOS ANDES: UN REFUGIO DE MEMORIA BIOCULTURAL MAPUCHE PEWENCHE
}

\section{Local knowledge in montane homegardens in the southern Andes: a refuge of Mapuche Pewenche biocultural memory}

\author{
Carla Marchant Santiago ${ }^{1 *}$, Nicolás Fuentes Acuña ${ }^{2}$, Santiago Kaulen Luks ${ }^{3} \&$ José Tomás Ibarra ${ }^{4}$ \\ ${ }^{1}$ Instituto de Ciencias Ambientales y Evolutivas, Universidad Austral de Chile. \\ ${ }^{2}$ Programa de Doctorado de Ciencias Sociales en Estudios Territoriales, Universidad de Los Lagos. \\ ${ }^{3}$ Escuela de Geografía, Universidad Austral de Chile. \\ ${ }^{4}$ Laboratorio ECOS (Ecología-Complejidad-Sociedad), Centro UC de Desarrollo Local \& Centro de Estudios Interculturales e Indígenas (CIIR), \\ Educación e Interculturalidad (CEDEL); Núcleo Milenio Centro para el Impacto Socioeconómico de las Políticas Ambientales (CESIEP) \\ \& Center of Applied Ecology and Sustainability (CAPES), Pontificia Universidad Católica de Chile.
}

\author{
Identificador ORCID de los autores y e.mail \\ Carla Marchant Santiago: https://orcid.org/0000-0002-4040-8372.E-mail: carla.marchant@uach.cl \\ Nicolás Fuentes Acuña: https://orcid.org/0000-0001-5376-1098. E-mail: nicolasricardoalberto.fuentes@alumnos.ulagos.cl \\ Santiago Kaulen Luks: https://orcid.org/0000-0002-7684-3237. E-mail: santiagokaulen@gmail.com \\ José Tomás Ibarra: https://orcid.org/0000-0002-7705-3974. E-mail: jtibarra@uc.cl \\ *Autor corresponsal
}

\begin{abstract}
Recibido: 02-06-2020. Aceptado: 03-08-2020. Fecha de publicación on-line: 02-10-2020
Citation / Cómo citar este artículo: Marchant Santiago, C., Fuentes Acuña, N., Kaulen Luks, S., Ibarra, J.T. (2020). Saberes locales en huertas de montaña del sur de los Andes: un refugio de memoria biocultural mapuche pewenche. Pirineos, 175, e060. https://doi.org/10.3989/pirineos.2020.175010

RESUMEN: Las huertas de montaña son sistemas socio-ecológicos clave para la producción agrícola destinada al consumo familiar. El cuidado y gestión de ellas recae principalmente en manos de mujeres que desarrollan diversas prácticas agrícolas que han aprendido y cultivado gracias a la transmisión de los saberes locales. Con un enfoque metodológico mixto, que incluyó fichas de caracterización predial, entrevistas semiestructuradas y grupos focales, este trabajo recopiló y sistematizó los principales saberes locales, asociados a conocimientos, prácticas y creencias, de un grupo de mujeres mapuche pewenche en una comuna cordillerana del sur de Chile. Los resultados permitieron distinguir tres categorías de saberes asociados al manejo de la huerta: i) control de organismos patógenos, ii) manejo y fertilidad del suelo, y iii) diseño y gestión de sistemas productivos. Estos saberes participan en la construcción de una memoria biocultural asociada a la huerta que ha perdurado en el tiempo y se ha transmitido entre generaciones. Evocar esta memoria permite mantener vivo el pasado, comprender el presente y planificar de manera sustentable el futuro de la agricultura a pequeña escala en territorio ancestral mapuche pewenche del sur de los Andes.
\end{abstract}


PALABRAS CLAVE: Agrobiodiversidad; Chile; huerta familiar; prácticas agrícolas; soberanía alimentaria.

\begin{abstract}
Montane homegardens are key socio-ecological systems for agricultural production and family consumption. Care and management lies mainly on the hands of women who developed diverse agricultural practices that they have learned and nurtured due to the transmission of local knowledge. With a mixed methodological approach, which included land property characterization, semi-structured interviews and focus groups, this work compiled and systematized the local wisdom, associated with knowledge, practices and beliefs, of mapuche-pewenche communities from southern Chile. We identified three categories of wisdom associated with the management of montane homegardens: i) control of pathogenic organisms, ii) fertility of the soil, and iii) design and stewardship of farm systems. This wisdom contributes to build a biocultural memory associated with homegardens that has endured over time and has been transmitted through generations. Recalling this memory allows maintaining the past alive, comprehend the present and sustainably shape the future of small-scale agriculture in ancestral Mapuche-Pewenche territory of the southern Andes.
\end{abstract}

KEY WORDS: Agrobiodiversity; Chile; homegardens; agricultural practices; food sovereignty.

\section{Introducción}

A nivel mundial, aproximadamente 1.400 millones de agricultores, incluyendo campesinos y comunidades indígenas, mantienen viva la agricultura familiar como un modelo productivo caracterizado por su complejidad, diversidad y resiliencia (Ibarra et al., 2020; Koohafkan \& Altieri, 2011), particularmente en sistemas socioecológicos de montaña (Marchant et al., 2019). Las huertas, definidas como un sistema espacialmente delimitado y ubicado cerca de la vivienda familiar, son parte fundamental de la agricultura familiar (FAO, 2014; Ibarra et al., 2019). En las huertas ocurren procesos continuos de domesticación, diversificación y producción agrícola asociados al cuidado y gestión de semillas, plantas herbáceas, arbustos, árboles y animales (Galluzzi et al., 2010). En este sentido, las huertas son vitales para la reproducción y transmisión intergeneracional de los saberes asociados a la producción de alimentos, la reproducción de la cultura local y la subsistencia de las familias campesinas más vulnerables (Landon-Lane, 2005; Calvet-Mir et al., 2011). Del mismo modo, las huertas albergan variedades $\mathrm{y}$ ecotipos tradicionales de plantas y animales en riesgo de desaparecer (Barthel et al., 2013). Las huertas se construyen a partir de lo colectivo y son fundamentales para el fortalecimiento de los vínculos sociales, transformándose en un espacio de memoria colectiva o "memoria biocultural" (Nazarea, 2006). La memoria biocultural se refiere a las "conciencias históricas comunitarias" o saberes acumulados a través del tiempo y espacio, que una identidad cultural concreta posee acerca del manejo de los territorios y sus elementos (Toledo \& Barrera-Bassols, 2008). Para entender la relación existente entre producción agraria y los procesos ecológicos y culturales propios de cada cultura y territorio, se evoca a la memoria de los individuos que lo habitan. La pérdida de agrobiodiversidad y del conjunto de conocimientos, prácticas y creencias asociadas a ella se ha asociado, entre otros, a la erosión de la memoria biocultural o a la "amnesia producida por los sistemas agroindustriales intensivos" (Toledo \& BarreraBassols, 2008; Urra \& Ibarra, 2018).
La modernización agraria impulsada por la Revolución Verde (1960-1980) en los países latinoamericanos, basada en una producción tecnificada a gran escala y altamente dependiente de insumos externos, desencadenó una serie de problemáticas para la agricultura familiar (Altieri et al., 2011), de las cuales el campo chileno no estuvo ajeno (Rosenblitt et al., 2001). Algunos efectos de este proceso fueron la invisibilización de saberes locales ligados a su mantención y desarrollo (Navarrete, 2017), junto con la creciente pérdida de la soberanía alimentaria local (López-García \& López-López, 2003). Esto último se tradujo en un mayor control externo de la producción agrícola local (Lozano, 1985), promoviendo cambios en las dinámicas de producción. Este proceso promovió también la erosión de las formas tradicionales de producción y manejo agrícola que las comunidades campesinas habían desarrollado a lo largo de su historia, incluyendo el cultivo de huertas familiares (Urra \& Ibarra, 2018). Además, se llegó incluso a la erosión de prácticas y saberes locales que a lo largo de generaciones las comunidades se encargaron de heredar (Toledo, 2003).

No obstante, autores de disciplinas como la Ecología Aplicada y la Antropología, han contribuido a teorizar, analizar, comprender y revitalizar los conocimientos y prácticas que integran estos saberes locales. Es así como Berkes et al. (2000:1252), se refieren al conocimiento ecológico local como un "cuerpo acumulativo de conocimientos (corpus), prácticas (praxis) y creencias (kos$m o s$ ), acerca de las relaciones de los seres vivos (incluidos los humanos) entre sí y con su medio ambiente, el que evoluciona a través de procesos adaptativos y es transmitido de una generación a otra”. Por otro lado, desde una perspectiva antropológica, Nazarea (2006) amplía esta definición, señalando que el conocimiento ecológico local son en realidad saberes locales que integran cuerpos de conocimiento, práctica y creencia pero que, a su vez, evocan una experiencia práctica vivida, siendo asociado de esta forma a emociones y memorias. De esta forma, los conocimientos locales sobre la agricultura, sus implicancias prácticas (producción) y el sistema subjetivo de creencias, están íntimamente relacio- 
nados en una memoria biocultural que modela pasado, presente y futuro. Por su parte, de acuerdo a Toledo \& Barrera-Bassols (2008), existirían distintos tipos de saberes, incluyendo los (i) astronómicos ligados a los tipos de astros; (ii) físicos que hacen alusión a los tipos de vientos, nubes y climas; (iii) biológicos en los que reconocen las plantas, animales y hongos asignándoles un uso determinado como la medicina y la alimentación; y (iv) ecogeográficos, donde se reconocen unidades de vegetación y paisajes, basándose en el relieve o en las estructuras geomorfológicas.

En este trabajo proponemos que las huertas son verdaderos "refugios" de memoria biocultural y que el conjunto de conocimientos, prácticas y creencias que se desarrollan en ellas reflejan los saberes locales, ya que por medio de la memoria las culturas indígenas heredan estos saberes (Barthel et al., 2013; Toledo, 2003). Ejemplos de estos refugios de memoria biocultural incluyen, por ejemplo, la chakra diversificada de Pastaza, en la Región Amazónica de Ecuador. En la chakra, los indígenas kichwa han transmitido sus saberes sobre la gestión espacial y temporal de la biodiversidad agrícola para reducir los riesgos de desabastecimiento y promover la soberanía alimentaria de su población (Alemán et al., 2015). Otro ejemplo corresponde a la reconocida práctica de camellones o waru waru, método agrícola tradicional de manejo del suelo y del agua, desarrollada desde tiempos prehispánicos en el altiplano andino para mitigar efectos de las heladas en cultivos (Lhomme \& Joinville Vacher, 2003). Estos ejemplos muestran cómo las prácticas representan la materialización del conocimiento a partir de la propia experiencia (Reckwitz, 2002). Por tanto, estas prácticas no pueden ser consideradas solo herramientas para la producción de alimentos, sino que también, son un reservorio de los saberes locales en la memoria biocultural.

Las huertas de montaña del sur de los Andes de Chile, son un territorio eminentemente femenino (Manosalva, 2017), ya que su gestión y el resguardo de los saberes ha recaído principalmente en mujeres campesinas, debido a la forma tradicional en que se distribuyen las actividades en el hogar (Barreau, 2014). Asimismo, las mujeres han debido resistir y gestionar la fuerte influencia de los programas de extensionismo estatal que han promovido la aplicación de paquetes tecnológicos convencionales (Marchant et al., 2019). Estos paquetes han contribuido a la homogenización de las prácticas agrícolas y sistemas alimentarios, la pérdida de cultivos tradicionales y la erosión de los saberes locales (Barreau et al., 2019). Sin embargo, las mujeres han logrado dar continuidad a los conocimientos y prácticas, generando alternativas tendentes al resguardo de la soberanía alimentaria (Barreau \& Ibarra, 2019).
Este trabajo busca responder las siguientes interrogantes: ¿Qué saberes locales de mujeres se identifican en el cuidado y gestión de las huertas? ¿Articulan estos saberes una memoria biocultural de las huertas de montaña? Para ello, el objetivo de este trabajo es identificar y sistematizar los saberes locales que se desarrollan en las huertas familiares de montaña en Kurarewe, territorio mapuche pewenche. También se discute sobre cómo estos saberes reflejan y mantienen viva la memoria biocultural, transformándose las huertas en verdaderos refugios de esta memoria en el sur de los Andes.

\section{Metodología}

\section{1. Área de estudio}

La investigación se desarrolló en la comuna de $\mathrm{Ku}$ rarewe, una zona montañosa en la Región de La Araucanía en el sur de Chile (Figura 1). La población rural comunal alcanza el $70 \%$ y un $50,3 \%$ de la población comunal se reconoce como mapuche pewenche (INE, 2017). El actual Plan de Desarrollo Comunal 2018-2022 promueve un desarrollo productivo del territorio con un enfoque de "soberanía alimentaria con identidad local, rescate de los conocimientos ancestrales y biodiversidad" (Ilustre Municipalidad de Kurarewe, 2018). Desde enero del 2018, Kurarewe junto a las comunas de Alto Biobío, Lonquimay y Melipeuco forma parte del proyecto "Red de Sistemas Importantes del Patrimonio Agrícola Nacional (SIPAN)"1 liderado por el Ministerio de Agricultura chileno y la FAO, el cual busca rescatar y valorizar el patrimonio agrícola y cultural del territorio, contribuyendo al desarrollo de una agricultura sustentable y resiliente que responda a los desafíos ambientales y que, además, promueva las economías locales (FAO, 2018).

\subsection{Diseño metodológico}

La metodología empleada tuvo un enfoque mixto. Se trabajó entre enero de 2017 y septiembre de 2019 con un grupo de 30 mujeres pertenecientes a la Cooperativa Zomo Ngen (espíritu de mujer en mapuzungun, lengua mapuche pewenche). Esta es una agrupación de mujeres campesinas mapuche y no mapuche que desarrollan la agricultura familiar, con actividades que incluyen el cultivo de huertas, complementado con la recolección de plantas medicinales, hongos comestibles $\mathrm{y}$ frutos silvestres.

\footnotetext{
${ }^{1}$ La red de sitios SIPAN que se está desarrollando en Chile, contempla además otros 3 sitios de interés en las siguientes regiones: General Lagos y Putre (Arica y Parinacota); Huara, Camiña, Colchane y Pica (Tarapacá), San Pedro de Atacama y Calama (Antofagasta). A estos se suma el archipiélago de Chiloé (Los Lagos) declarado como el primer sitio SIPAM de Chile en 2011 por sus paisajes, prácticas de cultivo de las más de 200 variedades locales de papas existentes y otros productos como el ajo chilote.
} 


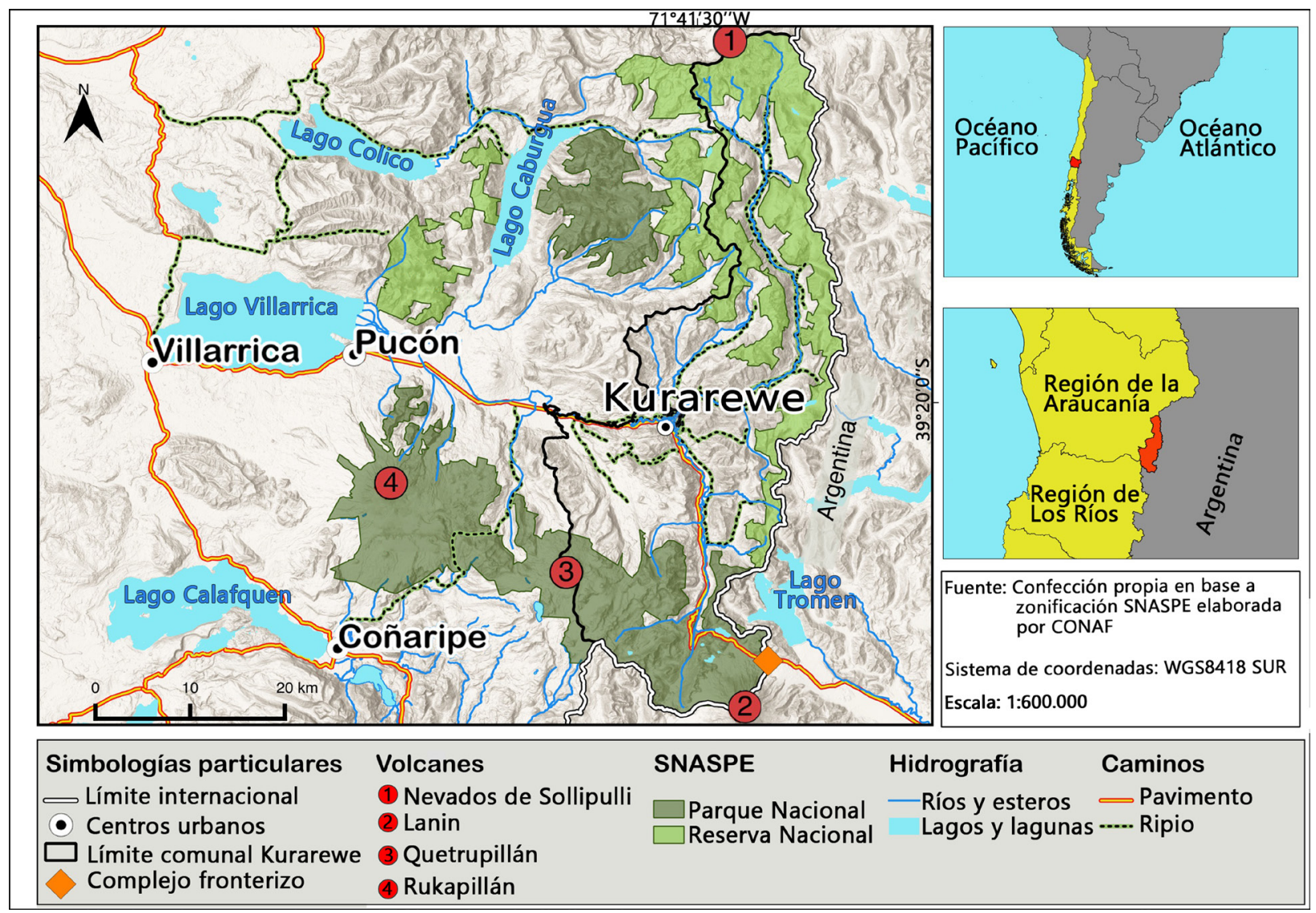

Figura 1. Mapa área de estudio. Fuente: Elaboración propia, 2019.

Figure 1. Study area.

\subsubsection{Toma de información}

Se realizaron 18 entrevistas semiestructuradas a las mujeres responsables de la huerta, con la finalidad de profundizar en los modos, las fuentes y formas de adquisición de los saberes asociados al manejo de las huertas. Además, se realizó una caracterización de los 18 predios de las entrevistadas, donde se incluyó la siguiente información: datos sociodemográficos de la familia, tipos de producción que se realiza en el predio (hortalizas, verduras, frutas, animales y productos de recolección), prácticas de manejo agrícola, estrategias de ordenamiento del predio e inventario de especies de plantas cultivadas intencionalmente a través de la identificación de su nombre común y especie (Schneider, 2010). También se incluyó información sobre recolección de especies vegetales y de hongos recolectados fuera del predio. Además, durante el proceso de investigación, se realizaron cinco grupos focales (conversatorios) de 2 horas de duración aproximada sobre diversas temáticas vinculadas al quehacer de las mujeres y sobre el territorio, a saber: identi- dad, vida cotidiana, recursos y bienes naturales, posibilidades de actividades turísticas, patrimonio local (Tabla 1). Estas instancias permitieron profundizar en temáticas vinculadas al tema central de la investigación, que eran claves para comprender mejor el contexto y también vislumbrar otros intereses y motivaciones de las mujeres con respecto a su actividad. Finalmente se realizó un registro fotográfico de las huertas visitadas (Newing, 2011).

\subsubsection{Sistematización y análisis de la información}

Las entrevistas realizadas ${ }^{2}$ fueron grabadas y transcritas para posteriormente realizar un análisis de contenido temático (Braun \& Clarke, 2006) utilizando el software Atlas.ti (2018). Lo anterior permitió organizar de manera inductiva la información y categorizar en tres grandes grupos los saberes locales: saberes para el control de organismos patógenos en los sistemas productivos, saberes locales para el manejo y fertilidad de suelos y saberes lo-

\footnotetext{
2 Las entrevistas y grupos focales cumplieron con los estándares de ética en investigación propuestos por Conicyt-Chile; para ello se contó con la firma de un consentimiento informado para participantes del proyecto.
} 
Tabla 1. Principales características de los grupos focales realizados.

Table 1 Main characteristics of Focus Groups.

\begin{tabular}{|l|l|l|l|}
\hline \multicolumn{1}{|c|}{ Conversatorio } & \multicolumn{1}{|c|}{ Fecha } & \multicolumn{1}{|c|}{ Temática/objetivo } & \multicolumn{1}{c|}{ Participantes } \\
\hline $\begin{array}{l}\text { Primer encuentro entre } \\
\text { investigadores y socias } \\
\text { de la Cooperativa }\end{array}$ & 12.2017 & $\begin{array}{l}\text { Presentar y compartir ideas, anhelos y alcances } \\
\text { del proyecto y la colaboración }\end{array}$ & $\begin{array}{l}\text { 22 socias, 3 miembros del equipo de } \\
\text { investigación, 1 invitado }\end{array}$ \\
\hline $\begin{array}{l}\text { Conociendo el territorio } \\
\text { de Kurarewe }\end{array}$ & 01.2018 & $\begin{array}{l}\text { Conversar sobre la historia local, y otros } \\
\text { elementos relevantes del espacio habitado por } \\
\text { las socias de la cooperativa }\end{array}$ & $\begin{array}{l}30 \text { socias, 2 miembros del equipo de } \\
\text { investigación, familia amiga de la } \\
\text { cooperativa (dos adultos y dos niños). }\end{array}$ \\
\hline $\begin{array}{l}\text { Identificando los } \\
\text { atractivos de nuestro } \\
\text { territorio }\end{array}$ & 06.2018 & $\begin{array}{l}\text { A través de un mapeo colectivo, identificar los } \\
\text { atractivos naturales y culturales del territorio }\end{array}$ & $\begin{array}{l}\text { 12 socias, 2 miembros del equipo de } \\
\text { investigación }\end{array}$ \\
\hline $\begin{array}{l}\text { Identificando } \\
\text { nuestro patrimonio } \\
\text { agroalimentario }\end{array}$ & 9.2018 & $\begin{array}{l}\text { Intercambiar conocimientos y experiencias sobre } \\
\text { el patrimonio agrícola de la comunidad }\end{array}$ & $\begin{array}{l}15 \text { socias, un miembro del equipo de } \\
\text { investigación y un invitado }\end{array}$ \\
\hline $\begin{array}{l}\text { La vida y lo cotidiano } \\
\text { en la huerta familiar }\end{array}$ & 8.2019 & $\begin{array}{l}\text { Profundizar sobre las experiencias de vida, } \\
\text { trabajo y saberes asociados a la huerta }\end{array}$ & $\begin{array}{l}15 \text { socias de la cooperativa y 1 miembro } \\
\text { del equipo de investigación }\end{array}$ \\
\hline
\end{tabular}

Fuente: elaboración propia

cales para el diseño y gestión de sistemas productivos en el predio.

Posteriormente, las prácticas asociadas a cada uno de los grupos anteriormente definidos, fueron clasificadas de acuerdo con la propuesta de Toledo \& Barrera-Bassols (2008), quienes distinguen cuatro categorías: astronómicos, físicos, biológicos y ecogeográficos, junto con cuatro dimensiones de los saberes: (i) estructurales (i.e. vinculadas a los elementos presentes en la naturaleza o taxonomías); (ii) dinámicas (i.e. asociación de patrones y procesos que ocurren en la naturaleza); (iii) relacionales (i.e. establece vínculos entre los elementos o los eventos naturales); y (iv) utilitarias (i.e. usos de los recursos naturales; Tabla 2).

Tabla 2. Sistematización de los diversos tipos de saberes locales. Table 2. Systematisation of different types of local knowledge.

\section{Resultados}

\subsection{Caracterización socioeconómica y productiva}

Las 18 mujeres entrevistadas en su predio eran en su totalidad oriundas del territorio y promediaban de 55 años; todas se identificaron como mapuche pewenche y eran responsables de la gestión de sus huertas. Con respecto a las dimensiones de los predios, estos promediaron 7,5 ha (rango 0,5 - 19 ha). Para la caracterización productiva, se realizó un inventario de plantas y hongos que incluyó plantas cultivadas intencionalmente en la huerta y/o predio y también las que se recolectan en el territorio. Este inventario permitió identificar un total de 123 especies (Anexo 1).

\begin{tabular}{|c|c|c|c|c|c|c|}
\hline Categorías & $\begin{array}{l}\text { Astronómicos } \\
\text { (AS) }\end{array}$ & \multicolumn{3}{|c|}{ Físicos (F) } & Biológicos (BI) & $\begin{array}{c}\text { Ecogeográficos } \\
\text { (EG) }\end{array}$ \\
\hline \multicolumn{2}{|c|}{ Dimensiones } & $\begin{array}{l}\text { Atmósfera } \\
\text { (AT) }\end{array}$ & $\begin{array}{l}\text { Litósfera } \\
\text { (LT) }\end{array}$ & $\begin{array}{l}\text { Hidrósfera } \\
\text { (HD) }\end{array}$ & & \\
\hline Estructural (E) & Tipos de astros & $\begin{array}{l}\text { Tipos de clima, } \\
\text { vientos, nubes, } \\
\text { periodos de } \\
\text { lluvia }\end{array}$ & $\begin{array}{l}\text { Unidades } \\
\text { de relieve, } \\
\text { rocas, } \\
\text { suelos }\end{array}$ & $\begin{array}{l}\text { Conocimiento } \\
\text { relativo a los } \\
\text { cursos de agua } \\
\text { superficiales y } \\
\text { subterráneos }\end{array}$ & $\begin{array}{l}\text { Conocimientos } \\
\text { sobre las plantas, } \\
\text { animales, hongos y } \\
\text { microorganismos }\end{array}$ & $\begin{array}{l}\text { Unidades de } \\
\text { vegetación y } \\
\text { paisaje }\end{array}$ \\
\hline Relacional (R) & \multicolumn{6}{|c|}{ Ligado a las relaciones entre los elementos o a los eventos naturales } \\
\hline Dinámico (D) & $\begin{array}{l}\text { Movimientos y } \\
\text { ciclos solares, } \\
\text { lunares, estelares }\end{array}$ & $\begin{array}{l}\text { Movimiento } \\
\text { de vientos y } \\
\text { nubes }\end{array}$ & $\begin{array}{l}\text { Erosión } \\
\text { de suelo y } \\
\text { otros }\end{array}$ & Ciclos de vida & $\begin{array}{l}\text { Movimientos del } \\
\text { agua }\end{array}$ & $\begin{array}{l}\text { Sucesión } \\
\text { ecológica }\end{array}$ \\
\hline Utilitario (U) & \multicolumn{6}{|c|}{ Sobre usos de recursos naturales y paisajes } \\
\hline
\end{tabular}

Fuente: modificado de Toledo y Barrera-Bassols (2008) 


\subsection{Saberes locales sobre huertas en Kurarewe}

En total se identificaron 19 prácticas reunidas en tres grandes grupos de saberes: 1. Saberes para el control de organismos patógenos, 2. Saberes sobre manejo y fertilidad del suelo, 3. Saberes sobre el diseño y gestión de sistemas productivos. A continuación, se describen las prácticas más frecuentemente mencionadas por las mujeres entrevistadas.

\subsubsection{Saberes locales para el control de organismos patógenos en los sistemas productivos}

Las participantes mencionaron diversas prácticas para enfrentar a plagas en sus sistemas productivos (Tabla 3 ). Aquellas mencionadas con mayor frecuencia fueron las siguientes:

i) Mantenimiento de policultivos (P): corresponde a la asociación de productos comestibles como verduras y hortalizas, con otras especies como flores, árboles, plantas aromáticas, entre otros. Según las agricultoras, esta práctica favorece la robustez de las plantas para combatir una plaga, debido a que aumenta la sinergia en el uso de nutrientes: "no se puede plantar solo de una planta, se van haciendo separaciones, pero hay que plantar de todo, entonces para que no se quiten la fuerza uno a otro" (Sector Los Sauces, 59 años). De esta forma el policultivo se transforma en una práctica permanente durante la estación agrícola, que contribuye en el control de plagas, ya sea por efecto de plantas trampa (plantas que atraen insectos y evitan la llegada a otras) o por medio de plantas aromáticas repelentes, tal como mencionan dos agricultoras: "mi suegra era una viejita muy de huerta. Ella siempre decía que uno tiene que tener de todo en la huerta... flores, hierbas medicinales, árboles, las verduritas y pastos. No hay que eliminar todo porque a veces llega un bicho que le gusta el pasto y como no encuentra, se va a la planta" (sector Pocolpén, 66 años). "Siempre hay que tener varias plantitas en el huerto y así se van los bichitos malos; como la ruda por ejemplo, porque es fuerte, el ajenjo blanco también” (sector Quiñenahuin, 70 años).

ii) Elaboración y uso de insecticidas/repelentes naturales (EIN): como por ejemplo la aplicación de cenizas en los cultivos donde, dependiendo del color, se reconocen distintos tipos de ceniza de mejor o peor calidad para este fin. Otras elaboraciones se hacen por medio de cocción de hierbas o partes de árboles nativos presentes en los predios, como menciona una de las entrevistadas: "agarro canelo, lo echo a hervir, hago agüita de canelo y lo empiezo a desparramar, y ligerito se va. Por lo menos el pilme cuando entra a las papas yo le echo pura agüita de canelo. También el laurel sirve" (sector Maichin Llafa, 63 años).

iii) Cultivo/cosecha tardía o temprana (CT): esta práctica se basa en el conocimiento biológico y temporal que las agricultoras poseen sobre las fechas de aparición de plagas. A modo de ejemplo, para controlar el Pilme (Epicauta pilme), una de las mujeres indica: "dicen que, si uno siembra a muy destiempo, el pilme cae en los cultivos y los mata porque la planta está muy chiquitita. Uno tiene que tratar de sembrar antes, en junio o julio, y ya está florecida la planta y el pilme no le hace nada” (sector Quiñenahuin, 41 años).

Tabla 3. Saberes locales para el control de organismos patógenos en los sistemas productivos.

Table 3. Local knowledge for the pathogenic organism in production systems.

\begin{tabular}{|c|c|c|c|c|}
\hline Propósito & Práctica & Descripción & Dimensión & Categoría \\
\hline \multirow{6}{*}{$\begin{array}{l}\text { Control de } \\
\text { organismos } \\
\text { patógenos }\end{array}$} & Policultivos (P) & $\begin{array}{l}\text { Práctica en la cual se cultivan dos o más especies vegetales que } \\
\text { se potencian y/o protegen entre sí. Incluye la incorporación } \\
\text { de flores y plantas ornamentales que por sus características } \\
\text { permiten repeler y/o concentrar en un lugar específico las plagas. }\end{array}$ & (E) (D) (U) & (BIO) \\
\hline & $\begin{array}{l}\text { Elaboración de } \\
\text { insecticidas naturales } \\
(\text { EIN) }\end{array}$ & $\begin{array}{l}\text { Preparación de "remedios caseros" en base a plantas, cortezas, } \\
\text { vegetales, frutos, etc. Incluye la aplicación de ceniza pre y } \\
\text { post cultivo de forma foliar o incorporándola al suelo, ya sea } \\
\text { disuelta en agua o en estado natural. }\end{array}$ & (U) (D) & (BIO) \\
\hline & $\begin{array}{l}\text { Cultivo/cosecha } \\
\text { tardía/temprana }(\mathrm{CT})\end{array}$ & $\begin{array}{l}\text { Sembrar/cosechar antes o después de sucesos climáticos y/o } \\
\text { biológicos que se conocen con anticipación. }\end{array}$ & (D) (R) & $\begin{array}{l}(\mathrm{AT}) \\
(\mathrm{BIO})\end{array}$ \\
\hline & $\begin{array}{l}\text { Postura de trampas } \\
\text { (PT) }\end{array}$ & $\begin{array}{l}\text { Elaboración de artefactos que permiten atraer y capturar } \\
\text { plagas. }\end{array}$ & (U) (D) & (BIO) \\
\hline & $\begin{array}{l}\text { Extracción manual } \\
\text { (EM) }\end{array}$ & $\begin{array}{l}\text { Consiste en extraer con las manos las plagas que así lo } \\
\text { permiten }\end{array}$ & (U) (R) & (BIO) \\
\hline & $\begin{array}{l}\text { Incorporación animal } \\
\text { (IA) }\end{array}$ & $\begin{array}{l}\text { Integración temporal de animales en los sistemas productivos } \\
\text { para que consuman las plagas. }\end{array}$ & (U) (D) & $\begin{array}{l}(\mathrm{BIO}) \\
(\mathrm{EG})\end{array}$ \\
\hline
\end{tabular}

Nomenclatura: Estructural (E), Relacional (R), Dinámico (D), Utilitario (U), Astronómicos (AS), Biológicos (BIO), Ecogeográficos (EG), Atmósfera (AT), Litósfera (LT), Hidrósfera (HD). Fuente: Elaboración propia 


\subsubsection{Saberes locales para el manejo de fertilidad del suelo}

La fertilidad de los suelos es esencial en la agricultura, dado que permite y sustenta la vida vegetal. Lograr suelos fértiles es uno de los desafíos a los que se enfrentan las mujeres huerteras al momento de iniciar sus procesos productivos. Se identificaron seis prácticas con esta finalidad (Tabla 4).

i) Reciclaje de desechos orgánicos (RD) y ii) incorporación de abonos orgánicos (IAO): ambas prácticas se orientan a nutrir el suelo y se realizan a través de la recolección de tierra de hoja que se recolecta en el bosque y también de materia orgánica, la cual es producida con elementos reciclados dentro del predio, con los que elaboran compost, guano, biopreparados y humus de lombriz. Así, los suelos se preparan para la siembra, tal como una de las mujeres señala: "líquidos le echo a las plantitas, es de los mismos galpones, son juguitos. Los remojo en un balde y le voy echando con vasito a cada planta. Se echa en un balde, le echa la mitad en agua, y la mitad de abono. Y se deja un día que se remoje eso, de ahi se revuelve bien y se le echa a cada planta un jarrito" (Sector Quiñenahuin, 56 años).

iii) Reconocimiento de suelos (RDS): se realiza a través de la observación directa, como relata una de las entrevistadas: "cuando la tierra está colorada, es que no está buena. Tiene que estar negrita para que sea buena tierra. Cuando está colorada hay que colocarle harto abono para que se arregle" (Sector Quiñenahuin, 40 años). Asimismo, se utiliza la experiencia sensorial para identificar no solo el color, sino también el olor, textura, e identificar plantas e insectos presentes en el suelo para determinar otras decisiones de planificación, como por ejemplo la adición de nutrientes al suelo, como explica una entrevistada: "cuando la tierra está rojiza, uno ve que le sale pura pinpinela, trunel y hasta hormigas...esto quiere decir que está muy lavada esa tierra" (Flor del Valle, 71 años).

iv) Cama alta de cultivo (CA): utilizado para preparar los suelos para la siembra. Este proceso se inicia con el volteo y picado de la tierra, ya sea con ayuda de animales (yunta de bueyes), herramientas manuales (pala, azadón, picota) o maquinaria motorizada (motocultivador). Posteriormente, se delimita un espacio generalmente rectangular sobre el cual se fabrica la cama alta, la cual en algunos casos es reforzada en su perímetro con tablas. El relleno de la cama se realiza incorporando capas de diferentes materiales, como abono animal (guano), restos vegetales (compost), en algunos casos humus de lombriz, paja o pastos secos, tierra de hojas y el sustrato volteado. Esto se hace hasta sobrepasar por unos $10 \mathrm{~cm}$ la superficie, logrando así un suelo esponjoso, profundo y nutrido.

v) Rotación de cultivos (RC) y vi) Movimiento de los sistemas productivos dentro del predio (MSP): la

Tabla 4. Saberes locales empleados para la fertilidad del suelo.

Table 4. Local knowledge used for soil fertility.

\begin{tabular}{|c|c|c|c|c|}
\hline Propósito & Práctica & Descripción & Dimensión & Categoría \\
\hline \multirow[t]{6}{*}{$\begin{array}{l}\text { Fertilidad } \\
\text { del suelo y } \\
\text { crecimiento } \\
\text { óptimo de } \\
\text { plantas }\end{array}$} & $\begin{array}{l}\text { Reciclaje } \\
\text { de desechos } \\
\text { orgánicos (RD) }\end{array}$ & $\begin{array}{l}\text { Consiste en la identificación y almacenaje de los } \\
\text { elementos que se utilizan para elaborar los abonos } \\
\text { orgánicos que son aplicados en los sistemas productivos. }\end{array}$ & (D) (U) & $(\mathrm{BIO})(\mathrm{EG})$ \\
\hline & $\begin{array}{l}\text { Incorporación de } \\
\text { abonos orgánicos } \\
\text { (IAO) }\end{array}$ & $\begin{array}{l}\text { Consiste en incorporar nutrientes de origen animal/vegetal } \\
\text { al suelo ya sea antes de la siembra o durante el crecimiento } \\
\text { de la planta. Se aprecia el uso de preparaciones como } \\
\text { compost, biopreparados, humus de lombriz, junto con la } \\
\text { incorporación directa de guano y paja. }\end{array}$ & (D) (U) & (LT) (BIO) \\
\hline & $\begin{array}{l}\text { Reconocimiento } \\
\text { de suelos (RDS) }\end{array}$ & $\begin{array}{l}\text { Distinción de la fertilidad del suelo en base a indicadores } \\
\text { físicos y biológicos. }\end{array}$ & (E) (R) (D) & (LT) (BIO) \\
\hline & $\begin{array}{l}\text { Rotación de } \\
\text { cultivos (RC) }\end{array}$ & $\begin{array}{l}\text { Se define como la sucesión recurrente y regular de } \\
\text { diferentes cultivos en el mismo terreno a lo largo de } \\
\text { varios años. }\end{array}$ & (R) (D) & $\begin{array}{l}(\mathrm{BIO})(\mathrm{LT}) \\
(\mathrm{EG})\end{array}$ \\
\hline & $\begin{array}{l}\text { Movimiento } \\
\text { de sistemas } \\
\text { productivos } \\
\text { (MSP) }\end{array}$ & $\begin{array}{l}\text { Consiste en cambiar de posición los sistemas } \\
\text { productivos dentro del predio con el fin de no agotar el } \\
\text { suelo y recuperarlo. }\end{array}$ & (R) (D) & $\begin{array}{l}(\mathrm{BIO})(\mathrm{LT}) \\
(\mathrm{EG})\end{array}$ \\
\hline & $\begin{array}{l}\text { Cama alta de } \\
\text { cultivo (CA) }\end{array}$ & $\begin{array}{l}\text { Consiste en la delimitación de un montículo cuadrado } \\
\text { o rectangular que se levanta } 30 \mathrm{~cm} \text { sobre el suelo. Se } \\
\text { construye cavando una zanja a la cual se le incorpora } \\
\text { paja, abono y tierra. }\end{array}$ & (U) & (LT) \\
\hline
\end{tabular}

Nomenclatura: Estructural (E), Relacional (R), Dinámico (D), Utilitario (U), Astronómicos (AS), Biológicos (BIO), Ecogeográficos (EG), Atmósfera (AT), Litósfera (LT), Hidrósfera (HD). Fuente: Elaboración propia 
primera refiere especialmente a las especies cultivadas en la huerta y la segunda principalmente a la modificación de los espacios donde hay animales (corrales, galpones, gallineros, entre otros; Figuras 2 y 3 ). Ambas prácticas se realizan para la mantención de la fertilidad del suelo, alternando cada 4 o 6 años los sistemas productivos dentro del predio. En algunos casos, la rotación se inicia con la huerta, la que se moviliza hacia donde se encontraba el corral, aprovechando el abono que fue incorporado naturalmente por los animales. El corral se construye donde estaba la huerta, comenzando nuevamente el proceso de fertilización del suelo, como explica una de las participantes "donde está mi huerta, era un corral de ovejas... pero muchos años no se puede tener en el mismo sector la huerta, hay que rotar dependiendo del espacio que esté mejor la tierra" (Sector Flor del valle, 58 años).
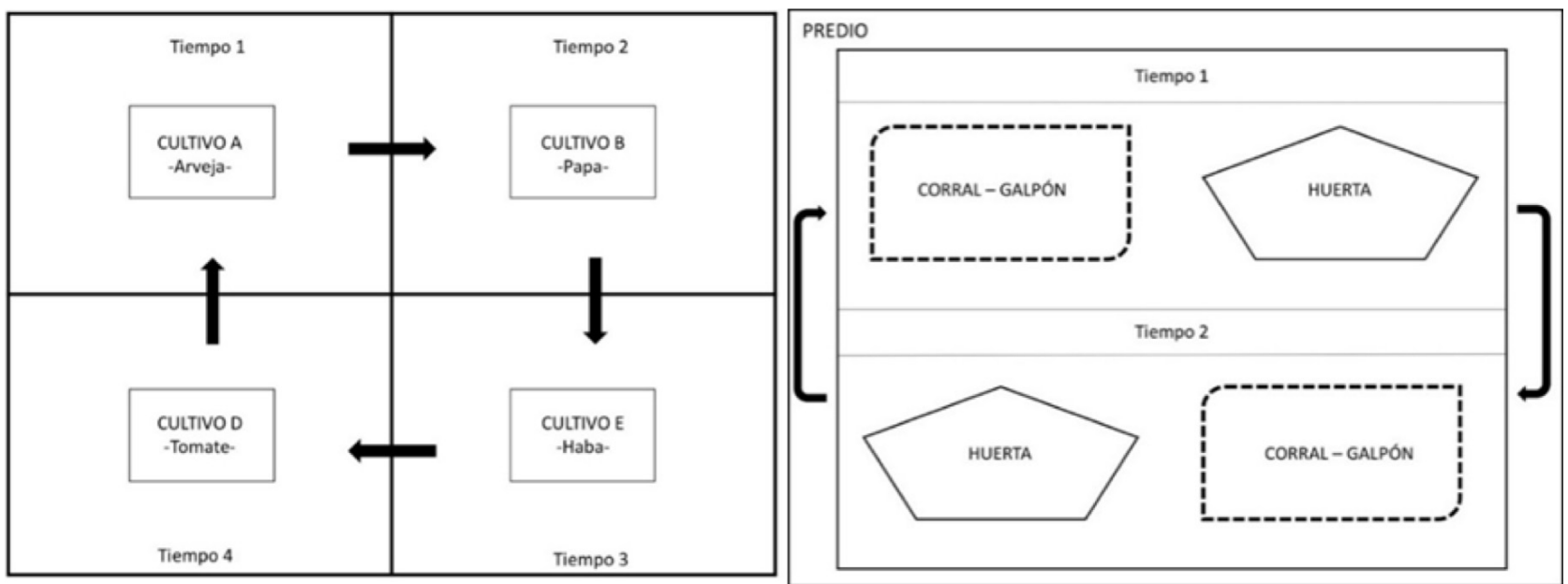

Figura 2. Rotación de cultivos en la huerta (izquierda) y en el predio (derecha). Fuente: Elaboración propia, 2019. Figure 2. Crop rotation on homegardens (left) and on the farm (right).



Figura 3. Planificación y preparación de cultivos y rotación en la huerta. Fuente: autores, 2020.

Figure 3. Planning, preparation of crops and rotation in homegardens. 


\subsubsection{Saberes para el diseño y gestión de sistemas productivos en el predio}

El mantenimiento de la diversidad fue identificada como un principio primordial en la gestión del predio y en la implementación de las prácticas productivas en la huerta. Esto se evidenció en el uso de más de una unidad ecogeográfica en el espacio productivo. Este principio se aplica tanto a nivel predial, como también sobre el espacio comunitario (bosques, cerros, praderas, caminos, ríos, entre otros). Lo anterior da como resultado la diversificación de estrategias productivas en el tiempo y el espacio. En este contexto, se identificaron distintas prácticas que las mujeres desarrollan con el objetivo de producir y mantener sistemas productivos diversos, tanto para el consumo doméstico como para la comercialización de excedentes (Tabla 5).

i) Ordenamiento del predio (OP): práctica que busca planificar el uso espacial y temporal de los recursos, localizar la infraestructura necesaria y definir las formas de producción más adecuadas. Para ello, se emplean conocimientos provenientes de la observación continua de la naturaleza, como el reconocimiento de las mejores horas de sol y la trayectoria diaria de este para determinar la orientación de huertas e invernaderos. También se planifica en función de que la huerta y la producción no se vean afectadas por los vientos de la cordillera (puelche) o ataques de fauna, como lo relata una de las entrevistadas: "la huerta va aquí porque aquí tenemos una huerta de manzana y es donde da el sol. El invernadero también. La bodega está ahí para no calentar mucho. Ahí tengo cocina a fuego y hago tortillas. El gallinero lo puse ahi para que proteja si viene el zorro, que no venga nada a agarrar en la noche cuando uno está durmiendo" (Puente basas chico, 56 años).

ii) Uso del calendario lunar (UCL): este es un saber que permite facilitar las prácticas de diseño y gestión de

Tabla 5. Saberes asociados al diseño, gestión y diversificación de sistemas productivos.

Table 5. Local knowledge associated with design, management, and diversification of production systems.

\begin{tabular}{|c|c|c|c|c|}
\hline Propósito & Práctica & Descripción & Dimensión & Categoría \\
\hline \multirow{6}{*}{$\begin{array}{l}\text { Diseño, } \\
\text { gestión y } \\
\text { diversificación } \\
\text { de sistemas } \\
\text { productivos }\end{array}$} & $\begin{array}{l}\text { Ordenamiento del } \\
\text { predio }(\mathbf{O P})\end{array}$ & $\begin{array}{l}\text { Corresponde a la delimitación de los espacios productivos } \\
\text { dentro del predio con el fín de aprovechar al máximo su } \\
\text { potencial. Aquí se articulan los saberes relacionados al } \\
\text { aprovechamiento de los cursos de agua, tipos de suelo, } \\
\text { movimiento del sol, percepción de riesgos, entre otros. }\end{array}$ & (E) (D) & $\begin{array}{l}\text { (HD) (LT) } \\
\text { (AS) }\end{array}$ \\
\hline & $\begin{array}{l}\text { Uso de calendario } \\
\text { lunar (UCL) }\end{array}$ & $\begin{array}{l}\text { Corresponde al uso de calendarios en base a las fases lunares } \\
\text { para controlar la programación de actividades agrícolas. }\end{array}$ & (D) & $(\mathrm{BIO})(\mathrm{AS})$ \\
\hline & $\begin{array}{l}\text { Producción, } \\
\text { guarda e } \\
\text { intercambio de } \\
\text { semillas (PGS) }\end{array}$ & $\begin{array}{l}\text { Es la selección de plantas específicas de una especie en el } \\
\text { sistema productivo (generalmente las más fuertes y sanas), las } \\
\text { cuales se dejan crecer hasta que produzcan semillas. Cuando } \\
\text { estas están maduras, se cosechan y guardan para ser utilizadas } \\
\text { en la próxima siembra. Destaca aquí su vinculación con el } \\
\text { trafkintü como una práctica cultural ancestral del pueblo } \\
\text { mapuche que consiste en el intercambio (sin mediación } \\
\text { de dinero) de propágulos (semillas, esquejes, etc.) y los } \\
\text { conocimientos asociados a estos, ya sea en un evento público } \\
\text { o en el mismo predio. }\end{array}$ & (E) (D) (U) & (BIO) \\
\hline & $\begin{array}{l}\text { Compartir los } \\
\text { saberes (CS) }\end{array}$ & $\begin{array}{l}\text { Consiste en el intercambio oral de experiencias prácticas } \\
\text { relacionadas a la cultura ancestral mapuche pewenche y } \\
\text { también campesina, donde existe una profunda relación entre } \\
\text { lo humano y lo natural, bajo elementos que conducen la } \\
\text { cotidianeidad y modo de vida en el territorio. }\end{array}$ & $\begin{array}{l}\text { (E) }(\mathrm{R}) \\
\text { (D) (U) }\end{array}$ & (EG) \\
\hline & $\begin{array}{l}\text { Mantención de } \\
\text { quintas (QTS) }\end{array}$ & $\begin{array}{l}\text { Corresponde a un espacio específico dentro del predio en el } \\
\text { cual se encuentran variedades de árboles frutales. }\end{array}$ & (E) & $\begin{array}{c}(\mathrm{BIO}) \\
(\mathrm{EG})\end{array}$ \\
\hline & $\begin{array}{l}\text { Crianza de } \\
\text { animales (CA) }\end{array}$ & $\begin{array}{l}\text { Corresponde a la reproducción y cuidado de animales con el } \\
\text { fin de sustentar las necesidades del grupo familiar. Existe una } \\
\text { estrecha relación entre esta actividad y la agrícola, ya que los } \\
\text { desechos producidos por los animales (guano) son la materia } \\
\text { prima de los abonos. Las lanas y cueros también son utilizados } \\
\text { para artesanías. }\end{array}$ & (E) (U) & (BIO) \\
\hline & $\begin{array}{l}\text { Recolección en el } \\
\text { bosque (REC) }\end{array}$ & $\begin{array}{l}\text { Se refiere a la recolección de productos fuera del predio, } \\
\text { generalmente en el bosque. Entre estos se encuentran } \\
\text { alimentos, materiales de construcción, material orgánico, } \\
\text { plantas medicinales y materia prima para artesanías. }\end{array}$ & $(\mathrm{E})(\mathrm{D})(\mathrm{U})$ & $\begin{array}{c}(\mathrm{BIO}) \\
(\mathrm{EG})\end{array}$ \\
\hline
\end{tabular}


la huerta. Algunas entrevistadas señalaron que consideran las fases lunares para la siembra y/o cosecha, demostrando la aplicación de un conocimiento astronómico y dinámico respecto al comportamiento de las plantas según la fase en la que se encuentra la luna. Una de las mujeres explica cómo organiza su trabajo: "la siembra es con menguante, o sea cuando la luna se está terminando, se ve poco... en esa sembramos nosotros" (Sector Flor del valle, 71 años). En este sentido, cabe destacar que las productoras son conscientes de que el calendario lunar influye directamente en la productividad de sus huertas.

iii) Producción, guarda e intercambio de semillas (PGS): corresponde a los procesos que permiten el cuidado, la regeneración y el mantenimiento de las especies cultivadas. Es una de las prácticas más relevantes para la subsistencia en el tiempo de las variedades tradicionales. Si bien la mayoría de las mujeres comparten el mismo tipo de semillas en sus huertas, existen algunas que están en mano de un reducido grupo, por lo cual su mantención corre peligro y es necesario el intercambio material pero también de los saberes asociados para su reproducción, hacia generaciones más jóvenes. En este sentido, cómo seleccionar semillas con potencial reproductivo, es un tema relevante. Una de las mujeres explica como hacerlo "las lechugas que se me subieron van a ser para guardar semillas. Por allá tengo poroto que también lo estoy esperando para guardar su semilla...uno sabe que está listo porque cuando está maduro, está más durito" (Sector Flor del Valle, 71 años). (Figura 4).

iv) Práctica de compartir los saberes (CS): estrechamente ligada con la práctica anterior (punto iii), se encuentra el compartir los saberes en sus diversas manifestaciones. En la cosmovisión mapuche, destaca el trafkintü (Figura 5), ceremonia que implica no solo el intercambio material de semillas, sino también el de saberes, "yo siempre mantengo las semillas que me daban mis padres en mi huerta, o las personas más adultas, las lamiegnes o ñañas de edad. Ellas son sabias y han ido guardando para sembrar al otro año. Entonces uno trata de hacer trafkintü con ellas, así conservamos la semilla y evitamos traer transgénico”. La misma agricultora enfatiza el carácter simbólico de esta práctica, "en los trafkintü hay personas que tienen mucha sabiduría ancestral de semillas... es una instancia en la cual lo que se dice tiene que ser real y sincero. Si uno dice tener una semilla originaria de un territorio, así debe ser, y que sea ojalá una semilla de calidad, saber de qué variedad es, de dónde salió, los cuidados que hay que entregarle; todos esos conocimientos los debe tener uno. Se hace con mucho respeto y el intercambio
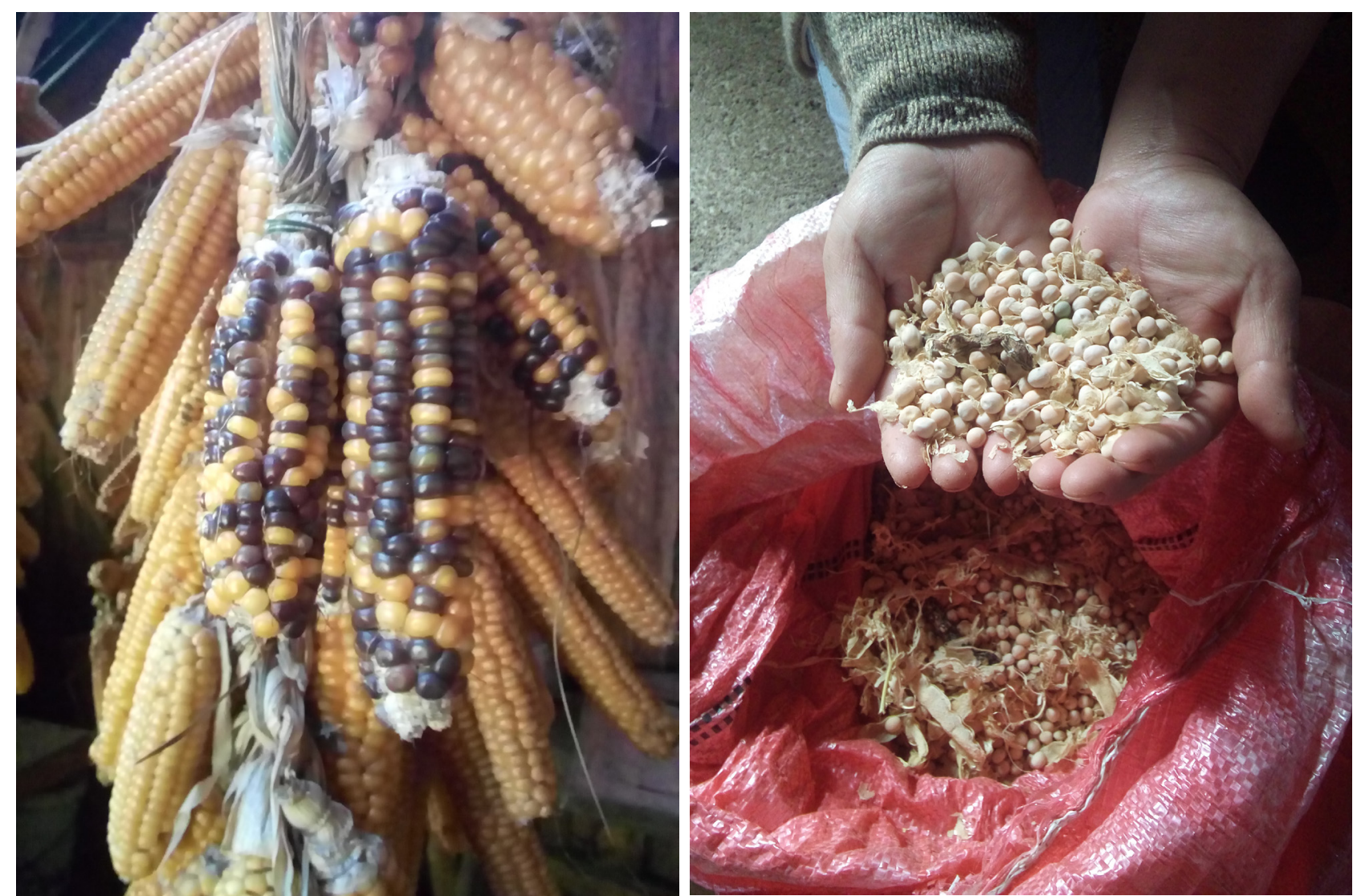

Figura 4. Selección de semillas para la guarda. A la izquierda maíz araucano (Zea mais) y a la derecha arvejas (Pisum sativum). Fuente: autores, 2019.

Figure 4. Selection of seeds for storage. On the left, Araucanian corn (Zea mais) and on the right, peas (Pisum sativum). 


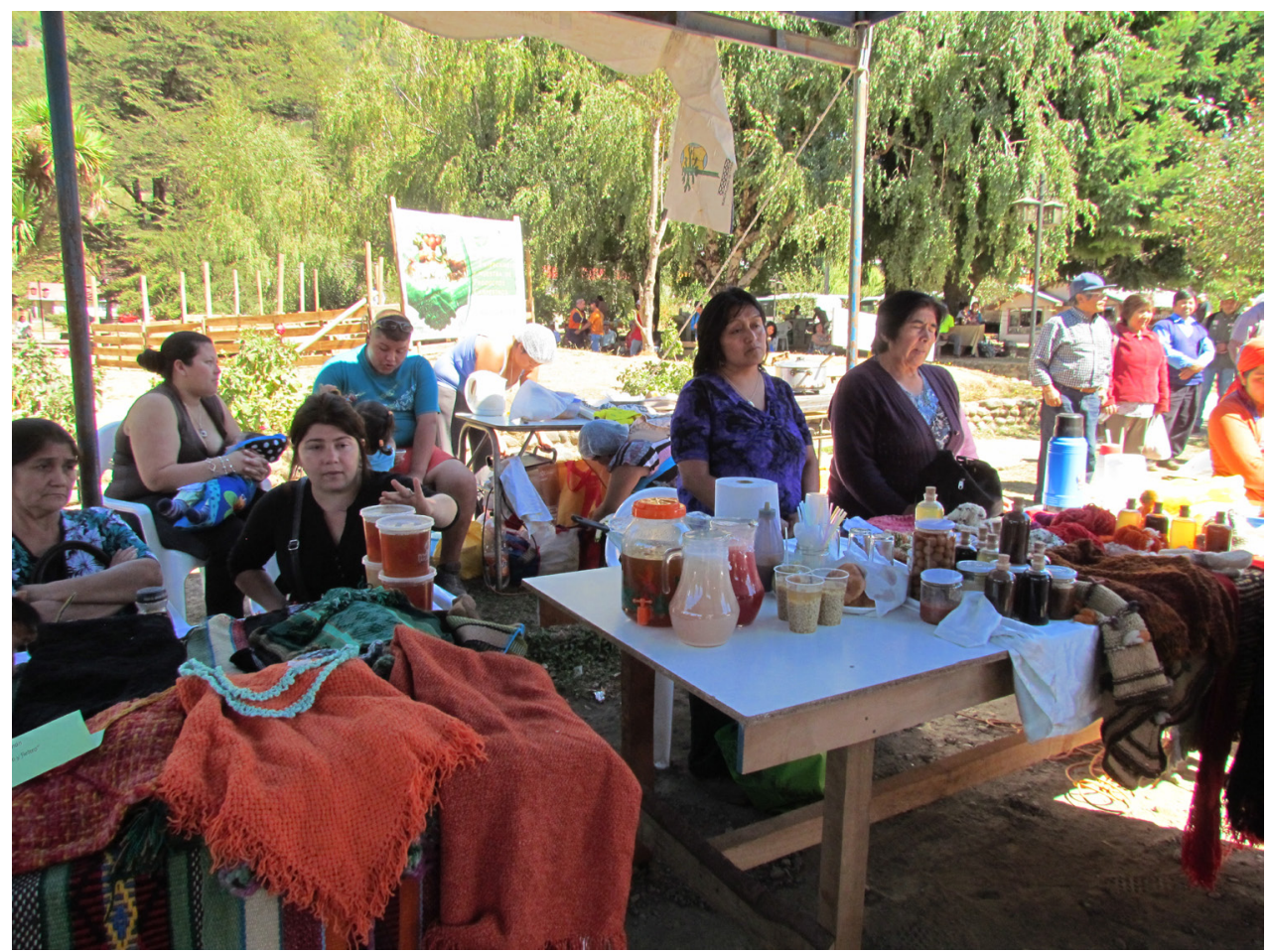

Figura 5. Celebración del trafkintü en Kurarewe, 2015. Fuente: autores, 2015.

Figure 5. Trafkintü celebration in Kurarewe, 2015.

tiene que ser con productos, nunca plata porque eso no es trafkintü”. (Chocol alto, 36 años).

Asimismo, el compartir en familia es también relevante; en instancias de la cotidianeidad se transmiten saberes relacionados con el territorio, los significados de la naturaleza y la propia forma de entender el mundo. Lo anterior se refleja en los siguientes relatos de tres entrevistadas al referirse, por ejemplo, a saberes climatológicos y de la naturaleza, propios de la montaña: "uno tuvo la suerte de criarse con los papás y compartir con los abuelos. Ellos decían que cuando las nubes van rápidas, se oscurece el cielo y se forma una bruma negra de nieblas, es que va a venir una lluvia". (Chocol alto, 36 años). "Los antiguos dicen que cuando hay cosas extrañas, como cuando florecen las quilas o manzanos en épocas que no corresponde, es porque va a venir un año de sequía o malo de alguna plaga" (Quiñenahuin, 77 años). "Cuando florecen las quilas, cuando florece el trébol, eso según el conocimiento de los viejitos dice que vendrá muy nevador... y las quilas significa que va a venir un tiempo muy malo y escasez de comida; la gente se prepara con los alimentos" (Quiñenahuin, 40 años).

\section{Discusión y reflexiones finales}

En este trabajo se identificaron y analizaron, apoyándose fundamentalmente en la voz de las participantes, diferentes prácticas asociadas al cultivo y gestión de huertas de montaña. Estos pequeños sistemas agrícolas constituyen verdaderos repertorios de experiencias y aprendizajes que las agricultoras han adquirido y acumulado a lo largo del tiempo y provienen del acervo cultural del territorio. En este contexto, la huerta como espacio de producción de alimentos, toma relevancia en la transmisión del conjunto de conocimientos, prácticas y creencias sobre la agricultura y el territorio (Chehuaicura et al., 2010; Marchant et al., 2019; Muñoz et al., 2019).

Los relatos dan cuenta que los saberes locales, en sus diversas categorías de conocimientos (astronómicos, físicos, biológicos o ecogeográficos) son experienciales y se construyen en la cotidianeidad del trabajo en la huerta, por ello son específicos y propios de cada territorio (De Walt, 1999). Los saberes locales identificados, no solo hacen referencia a las diversas prácticas ligadas a lo agrícola, sino que trascienden a otras dimensiones del quehacer en el territorio, especialmente en lo que respecta al fomento de la identidad local, la comprensión de la naturaleza en sus diversas manifestaciones y la importancia del compartir y trasmitir a los más jóvenes el valor de la ñuke тари (madre tierra). Lo anterior se relaciona con la racionalidad ambiental de los diversos pueblos indígenas, quienes establecen otras lógicas de vinculación entre el ser humano y la naturaleza (Reyes-García, 2009). Estos elementos pueden comprenderse como los articuladores de la memoria biocultural, la que se transmite en el tiempo y entre generaciones (Toledo \& Barrera-Bassols, 2008). 
Sin embargo, la migración de los más jóvenes a las ciudades y el poco interés por continuar con estas prácticas, son una amenaza que surge con fuerza. Existe evidencia de que en otros territorios de montaña, como Guipúzcoa del País Vasco, varias prácticas agrícolas locales se encuentran desapareciendo debido al envejecimiento de la población y el poco interés de las nuevas generaciones en mantener estos modos de vida (Alberdi, 2014). Estas prácticas también se encuentran amenazadas ya que los terrenos agrícolas están siendo reemplazados por segundas residencias, tal como ocurre en el valle del río Ter en el Pirineo catalán (Rigat et al., 2009). $\mathrm{Si}$ bien en el caso del territorio mapuche pewenche, existe un fuerte arraigo cultural sobre el territorio, en el que la transmisión de los saberes culturales está en estrecha relación con el entorno natural, la práctica agrícola y la espiritualidad (Quilaqueo \& Quintriqueo, 2010) es importante no perder de vista como estos procesos se están desarrollando.

Asimismo, existen otros procesos que facilitan la pérdida o erosión de los saberes locales y que van más allá de la actividad agrícola. Un ejemplo de ello es el régimen escolar que limita los tiempos del "enseñar haciendo" o la disminución del acceso a bosques para la recolección de frutos silvestres (Barreau et al., 2016). Esto es particularmente importante en el caso de prácticas como transmitir el conocimiento, lo que ocurre en la cotidianeidad del trabajo y la relación diaria entre niños y niñas, jóvenes y personas mayores. Asimismo, la homogeneización biocultural que actualmente afecta al sistema alimentario mapuche, dada la penetración de dietas comerciales en estaciones con baja productividad agrícola, genera una constante tensión en las posibilidades de mantención en el tiempo de los saberes locales descritos (Barreau et al., 2019).

Por otro lado, la mantención de las huertas por parte de las mujeres entrevistadas puede ser considerada como un acto político, que busca resistir los patrones de intervención de los espacios rurales y sus actividades por parte de programas estatales. En este sentido, la huerta es una potente herramienta para el resguardo de la soberanía alimentaria, entendida esta como un derecho de los pueblos a alimentos nutritivos y culturalmente apropiados producidos a través de métodos sostenibles y saludables (La Vía Campesina, 1996; Díaz \& Spiaggi, 2011). En este sentido, la acción de poder escoger cómo alimentar a la familia sin depender de factores externos es para las mujeres un hito relevante que permite a su vez el resguardo de los sistemas alimentarios y agrícolas locales (Barreau et al., 2019). Lo anterior queda reflejado en uno de los grupos focales, donde se discutieron estos aspectos y una mujer señaló que "la huerta es puro amor por las plantas...si siembras la huerta tienes que pensar en qué vas a producir y qué no vas a tener que andar comprando en otras partes. Además, lo que producimos en nuestras casas, en nuestros sectores, nuestra tierra, es todo sano, para uno, para nuestra familia y para quien venga. Cuando mis hijos se sientan a la mesa, ellos siempre están reconociendo que lo que se están sirviendo es del huerto, de ahí mismo de la casa, sea la carne, los huevos, la leche o todo lo que es verduras. Yo me quedé acá solamente para trabajar mi tierra, y hoy en día tengo todo sembrado" (Puente basas chico, 56 años).

Estas nociones de soberanía alimentaria mencionada por las agricultoras son particularmente importantes en las huertas de montaña; éstas representan un tipo de agricultura familiar que desafía una serie de dificultades para su desarrollo, tales como la escasez de tierras cultivables, la falta de conectividad y altos grados de intervención estatal (Fuentes \& Marchant, 2016). Asimismo, las huertas de montaña han resistido los efectos de la modernización agrícola que ha provocado tanto el aumento de la dependencia de tecnologías e insumos externos (Lozano, 1985), como la pérdida de variedades tradicionales (MartínezTorres \& Rosset, 2014). Esta resistencia es particularmente observable en el caso de la continuidad de la práctica del trafkintü en estos territorios aislados, la cual, a pesar de estos motores de cambio, ha permanecido en el tiempo y se consolida como una actividad trascendental para el intercambio de semillas, de otros productos agrícolas y de saberes locales.

La agricultura tradicional es clave en el resguardo de las semillas y en la mantención de la agrobiodiversidad a nivel mundial (Muñoz et al, 2019). Si bien este estudio no profundizó en este aspecto, cabe destacar el alto número de especies cultivadas intencionalmente en las huertas analizadas y también en las identificadas en el territorio circundante que se utilizan para distintos fines (alimentación, medicina, arte, etc.). Esto es relevante dado que, en otras zonas montañosas de la Península Ibérica, se ha descrito que existe una pérdida acelerada de cultivos tradicionales en las huertas familiares y de los conocimientos asociados a ellos (Rigat et al., 2009).

Finalmente, este trabajo contribuyó a generar nuevo conocimiento de temáticas relacionadas a los paisajes agroalimentarios de los Andes (Ibarra et al., 2020), a través de la identificación y comprensión de los saberes locales que cultivan la memoria biocultural en las huertas de montaña del sur de los Andes. Esta memoria debe comprenderse siempre como una articulación de conocimientos, prácticas y creencias situada y única de cada territorio. Por esta razón, estudios de caso como este nos permiten relevar su importancia y abogar por su revitalización y resguardo.

\section{Agradecimientos}

Los autores agradecen a las socias de la cooperativa, a Antonio Fernández y Patricia Araya por su valiosa cooperación durante esta investigación. Asimismo, agradecer a CONICYT por el financiamiento otorgado a través de los proyectos Fondecyt Iniciación N¹1140493 y ANID/Fondecyt Regular 1200291. JTI agradece el apoyo de ANID/ REDES (190033), ANID/FONDAP/15110006, ANID PIA/BASAL FB0002 y a CESIEP de la Iniciativa Científica Milenio del Ministerio de Economía, Fomento y Turismo (Chile). 


\section{Referencias}

Alberdi, J., 2014. Informe Técnico $\mathrm{N}^{\circ} 115$, Desarrollo Rural en el País Vasco: del programa de Agricultura de Montaña a las Asociaciones de Desarrollo Rural. Administración de la Comunidad Autónoma del País Vasco Departamento de Desarrollo Económico y Competitividad, 249pp. Vitoria-Gasteiz.

Alemán, R., Freire, J., \& Iparraguirre, M., 2015. Rescate de las prácticas agrícolas ancestrales y agricultura tradicional campesina para promover la producción sostenible a nivel familiar: la Chacra diversificada del CIPCA. Huellas del Sumaco Revista socioambiental de la Amazonía Ecuatoriana, 14: 6-11.

Altieri, M., Funes-Monzote, F., \& Petersen, P., 2011. Agroecologically efficient agricultural systems for smallholder farmers: contributions to food sovereignty. Agronomy for Sustainable Development, 32: 1-13. https://doi.org/10.1007/ s13593-011-0065-6

Barreau, A., 2014. Narrating changing foodways: wild edible plant knowledge and traditional food systems in Mapuche lands of the Andean Temperate Forests, Chile. Master thesis, Faculty of Forestry, University of British Columbia, Vancouver.

Barreau, A., Ibarra, J.T., Wyndham, F.S., Rojas, A. \&. Kozak. R.A., 2016. How can we teach our children if we cannot access the forest? Generational change in Mapuche knowledge of wild edible plants in Andean temperate ecosystems of Chile. Journal of Ethnobiology, 36(2): 412432. https://doi.org/10.2993/0278-0771-36.2.412

Barreau, A., Ibarra, J.T., Wyndham, F.S., Rojas, A., \& Kozak, R.A., 2019. Shifts in Mapuche food systems in southern Andean forest landscapes: historical processes and current trends of biocultural homogenization. Mountain Research and Development, 39(1): 12-23. https://doi.org/10.1659/ MRD-JOURNAL-D-18-00015.1

Barreau, A., \& Ibarra, M., 2019. Mujeres Mapuche y huertas andinas: espacios de fertilidad, soberanía y transmisión de saberes. (127-137 pp). En: Ibarra, J.T., Caviedes, J., Barreau, A., \& Pessa, N., (Eds.). 2019. Huertas familiares y comunitarias: cultivando soberanía alimentaria. Ediciones Universidad Católica de Chile, 288 pp. Santiago de Chile. https:// doi.org/10.2307/j.ctvkrkkbx.15

Barthel, S., Crumley, C., \& Svedin, U., 2013. Bio-Cultural Refugia - Safeguarding Diversity of Practices For Food Security and Biodiversity. Global Environmental Change, 23(5): $1142-1152$ gloenvcha.2013.05.001

Berkes, F., Colding, J. \& Folke, C., 2000. Rediscovery of traditional ecological knowledge as adaptive management. Ecological Applications, 10: 1251-1262. https://doi.org/10.1890/10510761(2000)010[1251:ROTEKA]2.0.CO;2

Braun, V. \& Clarke, V., 2006. Using thematic analysis in psychology. Qualitative Research in Psychology, 3(2): 77 101. doi:10.1191/1478088706qp063oa

Calvet-Mir, L., Calvet-Mir, M., Vaqué-Nuñez, L. \& Reyes-García, V., 2011. Landraces in situ Conservation: A Case Study in High-Mountain Home Gardens in Vall Fosca, Catalan Pyrenees, Iberian Peninsula. Economic Botany, 65(2): 146157. https://doi.org/10.1007/s12231-011-9156-1

Chehuaicura, N., Thomet, M. \& Perez, I., 2010. Identificación de criterios utilizados por especialistas tradicionales en la adaptación de la biodiversidad local en comunidades mapuche, región de la Araucanía (Chile). ISDA, 10pp. Montpellier, Francia.

De Walt, B., 1999. Combining indigenous and scientific knowledge to improved agriculture and natural resource management in Latin America. In: Pichon, F., Uquillas, J. \&
Frenchione, J. (Eds.) (1999). Traditional and Modern Natural Resource Management in Latin America. University of Pittsburgh Press, Pittsburgh, U.S.A. pp: 101-121.

Díaz, C. \& Spiaggi, E., 2011. Desarrollo rural, soberanía y seguridad alimentaria. Editorial de la Universidad Nacional de Rosario; FODEPAL - Núcleo de capacitación en Políticas Públicas FAO/RALC. Observatorio del Sur - UNR.

FAO, 2014. El Legado del AIAF 2014 y el camino a seguir. Acto de clausura Año Internacional de la Agricultura Familiar. Filipinas, FAO.

FAO, 2018. Sistemas importantes del patrimonio agrícola mundial: La biodiversidad agrícola y los ecosistemas resilientes. Prácticas agrícolas tradicionales e identidad cultural. Organización de las Naciones Unidas para la Alimentación y la Agricultura, 48pp. Recuperado de http://www.fao.org/3/ i9187es/I9187ES.pdf

Fuentes, N. \& Marchant, C., 2016. ¿Contribuyen las prácticas agroecológicas a la sustentabilidad de la agricultura familiar de montaña? El caso de Curarrehue, región de la Araucanía, Chile. Cuadernos de Desarrollo Rural, 13(78): 35-66. https://doi.org/10.11144/Javeriana.cdr13-78.cpas

Galluzzi, G., Eyzaguirre, P. \& Negri, V., 2010. Home gardens: neglected hotspots of agro-biodiversity and cultural diversity. Biodiversity and Conservation, 19(13): 4-12. https://doi. org/10.1007/s10531-010-9919-5

Ibarra, J.T., Barreau, A., Marchant, C., González, J.A., Oliva, M., Donoso-Correa, M.E., Antaki, B., Monterrubio-Solís, C. \& Sarmiento, F.O., 2020. Montology: an integrative understanding of mountain foodscapes for strengthening food sovereignty in the Andes. Ch. 24. In: Sarmiento, F.O. \& Frolich, L., (Eds.) The Elgar companion to geography, transdisciplinarity and sustainability. Edward Elgar Publishing, 391-405 pp. Cheltenham, U.K. https://doi. org/10.4337/9781786430106.00034

Ibarra, J.T., Barreau, A., Caviedes, J., Pessa, N. \& Urra, R., 2019. Huertas familiares tradicionales y emergentes: biodiversidad, aprendizaje y soberanía desde la interculturalidad (140-165 pp). En: Ibarra, J.T., Caviedes, J., Barreau, N. \& Pessa, N., (Eds.), 2019. Huertas familiares y comunitarias: cultivando soberanía alimentaria. Ediciones Universidad Católica de Chile. 288 pp. Santiago de Chile. https://doi. org/10.2307/j.ctvkrkkbx.16

Ilustre Municipalidad de Kurarewe, 2018. Actualización Plan de Desarrollo Comunal PLADECO 2018-2022. Kurarewe. Municipalidad de Kurarewe.

INE., 2017. XIX Censo Nacional de Población y VIII de Vivienda o Censo de Población y Vivienda.

Koohafkan, P. \& Altieri, M., 2011. Sistemas Ingeniosos del Patrimonio Agrícola Mundial un Legado para el Futuro. Organización de las Naciones Unidas para la Agricultura y la Alimentación, 41pp. Roma.

La Vía Campesina, 1996. Declaración de Tlaxcala de La Vía Campesina. II Conferencia Internacional De Tlaxcala. Recuperado de: https://viacampesina.org/es/ ii-conferencia-internacional-de-la-via-campesina-tlaxcalamexique-18-al-21-abril-1996/

Landon-Lane, C., 2005. Los medios de vida crecen en los huertos. Diversificación de los ingresos rurales mediante las huertas familiares. Organización de las Naciones Unidas para la Agricultura y la Alimentación, 62pp. Roma.

Lhomme, J.P. \& Vacher, J.J., 2003. La mitigación de heladas en los camellones del altiplano andino. Bulletin de l'Institut Français d'Études Andines, 32 (2): 377-399. https://doi. org/10.4000/bifea. 6556

López García, D., \& López López, J., 2003. Con la comida no se juega. 240pp., Madrid.

Lozano, W., 1985. Proletarización y campesinado en el capitalismo agroexportador. 144pp., Santo Domingo. 
Manosalva, H., 2017. Conocimientos y construcciones sobre la naturaleza en la huerta mapuche: estudio de caso con horticultoras y horticultores mapuche de la zona norte de Tirúa. Sustentabilidad(es) 8(16):3-45. Recuperado de: Conocimientos http://www.sustentabilidades.usach.cl/sites/sustentable/files/paginas/1._manosalva._final.pdf

Marchant, C., Fuentes, N. \& Castet, $\bar{G}$., 2019. Huertas de montaña: prácticas agroecológicas en la agricultura familiar de la Araucanía Andina (114-125 pp). En: Ibarra, J.T., Caviedes, J., Barreau, A. \& Pessa, N., (Eds.), 2019. Huertas familiares y comunitarias: cultivando soberanía alimentaria. Ediciones Universidad Católica de Chile, 288 pp. Santiago de Chile. https://doi.org/10.2307/j.ctvkrkkbx.14

Martínez-Torres, M.E. \& Rosset, P. 2014. Diálogo de saberes in La Vía Campesina: Food sovereignty and agroecology. Journal of Peasant Studies, 41(6): 979-97. doi:10.1080/030 66150.2013.872632.

Muñoz, A., Albornoz, F., \& Renwick, L., 2019. Agrobiodiversidad nativa ligada a pueblos indígenas y campesinos en Chile. En: Ibarra, J.T., Caviedes, J., Barreau, A. \& Pessa, N., (Eds.), 2019. Huertas familiares y comunitarias: cultivando soberanía alimentaria. Ediciones Universidad Católica de Chile. 288 pp. Santiago de Chile.

Navarrete, D., 2017. Transformaciones en la economía campesina por la instalación de la agroindustria en la comuna de Cabildo, provincia del Petorca. Tesis de Licenciatura Inédita. Universidad Academia de Humanismo Cristiano.

Nazarea, V., 2006. Local Knowledge and Memory in Biodiversity Conservation. The Annual Review of Anthropology, 35: 317-35. anthro.35.081705.123252

Newing, H., 2011. Conducting Research in Conservation: A Social Science Perspective. Routledge, 376 pp. New York, U.S.A. https://doi.org/10.1016/j.biocon.2012.06.001

Quilaqueo, D. \& Quintriqueo, S., 2010. Saberes educativos mapuches: un análisis desde la perspectiva de los kimches.
Revista Polis, 9(26): 337-360. http://dx.doi.org/10.4067/ S0718-65682010000200016

Reckwitz, A., 2002. Toward a theory of social practices. A development in culturalist theorizing. European Journal of Social Theory, 5(2): 243-263. https://doi.org/10.1177/ 13684310222225432

Reyes-García, V., 2009. Conocimiento ecológico tradicional para la conservación: dinámicas y conflictos. Papeles, 107: 39-55.

Rigat, M., Gartnatje, T. \& Valles, J., 2009. Estudio etnobotánico del alto valle del río Ter (Pirineo catalán): resultados preliminares sobre la biodiversidad de los huertos familiares. En: Llamas, F. \& Acedo, C. (Coordinadores): Botánica pirenaico-cantábrica en el siglo XXI. Ediciones Universidad de León, 739 pp., León, España.

Rosenblitt, J., Correa, M. \& Hajek, E.R., 2001. La modernización de la agricultura chilena. Pobreza y medio ambiente después de la reestructuración productiva. Mapocho, 50: 163-192.

Schneider, J., 2010. Toward an analysis of home-garden cultures: on the use of socio-cultural variables in home garden studies. In: Eyzaguirre, P.B. \& Linares, O.F. (Eds.), Home gardens and agrobiodiversity. Smithsonian Books, 41-55 pp Washington, DC, USA.

Toledo, V.M., 2003. Ecología, espiritualidad y conocimientode la sociedad del riesgo a la sociedad sustentable. Universidad Iberoamericana y Programa de Naciones Unidas para el Medio Ambiente, 146 pp, México.

Toledo, V.M., \& Barrera-Bassols, N., 2008. La memoria biocultural: la importancia ecológica de las sabidurías tradicionales. Icaria editorial, $232 \mathrm{pp}$. Barcelona.

Urra, R. \& Ibarra, J.T., 2018. Estado del conocimiento sobre huertas familiares en chile: agrobiodiversidad y cultura en un mismo espacio. Revista Etnobiología, 16(1): 31-46. Recuperado de: https://repositorio.uc.cl/handle/11534/ 33318

Anexo 1. Especies intencionalmente cultivadas y/o recolectadas por mujeres agricultoras mapuche pewenche del sur de los Andes. Annex 1. Species intentionally cultivated and / or collected by interviewed women.

\begin{tabular}{|l|l|l|}
\hline \multicolumn{2}{|c|}{ Familia } & \multicolumn{2}{|c|}{ Nombre común } & Sambucus nigra \\
\hline \multirow{5}{*}{ Adoxaceae } & Sauco*ntífico \\
\hline \multirow{5}{*}{ Amaranthaceae } & Acelga & Beta vulgaris var. cicla \\
\cline { 2 - 3 } & Betarraga & Beta vulgaris \\
\cline { 2 - 3 } & Espinacas & Spinacia oleracea \\
\cline { 2 - 3 } & Kinwa & Chenopodium quinoa \\
\hline \multirow{5}{*}{ Amaryllidaceae } & Ajo & Allium sativum \\
\cline { 2 - 3 } & Ajo chilote & Allium ampeloprasum \\
\cline { 2 - 3 } & Cebolla & Allium cepa \\
\cline { 2 - 3 } & Chalota & Allium ascalonicum \\
\cline { 2 - 3 } & Ciboulette & Allium schoenoprasum \\
\cline { 2 - 3 } & Puerro & Allium ampeloprasum var. porrum \\
\hline \multirow{5}{*}{ Apiaceae } & Cilantro & Coriandrum sativum \\
\cline { 2 - 3 } & Hinojo** & Foeniculum vulgare \\
\cline { 2 - 3 } & Perejil & Petroselinum crispum \\
\cline { 2 - 3 } & Zanahoria & Daucus carota \\
\hline Araliaceae & Sauco del diablo* & \\
\hline
\end{tabular}




\begin{tabular}{|c|c|c|}
\hline Familia & Nombre común & Nombre científico \\
\hline Araucariaceae & Araucaria/Pewén* & Araucaria araucana \\
\hline \multirow{8}{*}{ Asteraceae } & Ajenjo** & Artemisia absinthium \\
\hline & Alcachofa & Cynara scolymus \\
\hline & Bailahuén* & Haplopappus angustifolius \\
\hline & Éter** & Artemisia abrotarum \\
\hline & Lechuga & Lactuca sativa \\
\hline & Manzanilla** & Matricaria chamomilla \\
\hline & Papa chira/Topinambur & Helianthus tuberosus \\
\hline & Tusílago** & Petasites fragans \\
\hline \multirow{2}{*}{ Berberidaceae } & Michay del zorro* & Berberis linearifolia \\
\hline & Michay/Calafate* & Berberis rotundifolia \\
\hline Betulaceae & Abedul* & Betula spp \\
\hline Boletaceae & Loyo* & Boletus loyo \\
\hline \multirow{2}{*}{ Boraginaceae } & Borraja* & Borago officinalis \\
\hline & Consuelda & Symphytum officinale \\
\hline \multirow{2}{*}{ Brassicaceae } & Nabo & Brassica rapa subsp. rapa \\
\hline & Repollo & Brassica oleracea \\
\hline Bromeliaceae & Barba del abuelo* & Tillandsia usneoides \\
\hline Cactaceae & San pedro* & Echinopsis pachanoi \\
\hline Celastraceae & Maitén* & Maytenus boaria \\
\hline Chenopodiaceae & Paico* & Chenopodium ambrosioides \\
\hline Clavariaceae & Changle* & Ramaria flava \\
\hline \multirow{4}{*}{ Cucurbitaceae } & Alcayota & Cucurbita ficifolia \\
\hline & Pepino & Cucumis sativus \\
\hline & Zapallo de guarda & Curcubita maxima \\
\hline & Zapallo italiano & Curcubita pepo \\
\hline Cunoniaceae & Palo santo* & Weinmannia trichosperma \\
\hline \multirow{2}{*}{ Cyttariaceae } & Digüeñe* & Cyttaria espinosae \\
\hline & Lau llau* & Cyttaria harioti \\
\hline Elaeocarpaceae & Maqui** & Aristotelia chilensis \\
\hline Ephedraceae & Pingo-Pingo* & Ephedra chilensis \\
\hline Equisetaceae & Limpia de plata* & Equisetum bogotense \\
\hline \multirow{6}{*}{ Fabaceae } & Arveja & Pisum sativum \\
\hline & Culén* & Psoralea glandulosa \\
\hline & Habas & Vicia faba \\
\hline & Paramela* & Adesmia emarginata \\
\hline & Poroto & Phaseolus vulgaris \\
\hline & Poroto pallar & Phaseolus coccineus \\
\hline Fagaceae & Castaña & Castanea sativa \\
\hline Gentianaceae & Kachan lawen* & Centaurium cachanlahuen \\
\hline \multirow{2}{*}{ Grossulariaceae } & corinto, Zarzaparrilla* & Ribes rubrum \\
\hline & Grosella & Ribes uva-crispa \\
\hline Gunneraceae & Nalca* & Gunnera tinctoria \\
\hline
\end{tabular}


$16 \bullet$ C. MARCHANT SANTIAGO, N. FUENTES ACUÑA, S. KAULEN LUKS \& J. T. IBARRA

\begin{tabular}{|c|c|c|}
\hline Familia & Nombre común & Nombre científico \\
\hline Helvellaceae & Chicharrón de montaña* & Gyromitra antarctica \\
\hline Hydnaceae & Lengua de vaca* & Hydnum repandum \\
\hline Juglandaceae & Nogal & Juglans regia \\
\hline \multirow{11}{*}{ Lamiaceae } & Albahaca & Ocimum basilicum \\
\hline & Alhucema & Lavandula angustifolia \\
\hline & Chascú & Thymus vulgaris \\
\hline & Hierba buena** & Mentha spicata \\
\hline & Melisa** & Melissa officinalis \\
\hline & Menta & Mentha piperita \\
\hline & Orégano & Origanum majorana \\
\hline & Orégano & Origanum vulgare \\
\hline & Poleo** & Mentha pulegium \\
\hline & Salvia & sphacele salviae \\
\hline & Toronjil cuyano** & Marrubium vulgare \\
\hline \multirow{2}{*}{ Lauraceae } & Lingue* & Persea lingue \\
\hline & Peumo* & Cryptocarya alba \\
\hline Linaceae & Ñancolahuén* & Linum chamissonis \\
\hline Malvaceae & Tilo* & Tilia $s p$ \\
\hline Meripilaceae & Gar-gal* & Grifola gargal \\
\hline Morchellaceae & Morchella* & Morchella conica \\
\hline \multirow{3}{*}{ Myrtaceae } & Arrayán* & Luma apiculata \\
\hline & Cauchao* & Amomyrtus Luma \\
\hline & Murta* & Ugni candollei \\
\hline \multirow{3}{*}{ Nothofagaceae } & Coihuelawen/Coigüe* & Nothofagus dombeyi \\
\hline & Hualle/Pellín** & Nothofagus obliqua \\
\hline & Rauli* & Nothofagus alpina \\
\hline Onagraceae & Chilco* & Fuchsia magellanica \\
\hline \multirow{2}{*}{ Oxalidaceae } & Culle colorado* & Oxalis rosea \\
\hline & Vinagrillo* & Oxalis arenaria \\
\hline Plantaginaceae & Llantén* & Plantago major \\
\hline \multirow{4}{*}{ Poaceae } & Quila* & Chusquea quila \\
\hline & Avena & Avena sativa \\
\hline & Maíz & Zea mays \\
\hline & Trigo & Triticum aestivum \\
\hline Podocarpaceae & Lleuque* & Prumnopitys andina \\
\hline Polygonaceae & Sanguinaria* & Polygonum aviculare \\
\hline \multirow{2}{*}{ Proteaceae } & Avellana* & Gevuina avellana \\
\hline & Radal* & Lomatia hirusta \\
\hline \multirow{6}{*}{ Rosaceae } & Albaricoque & Prunus armeniaca \\
\hline & Cerezo & Prunus avium \\
\hline & Ciruelo & Prunus domestica \\
\hline & Durazno & Prunus persica \\
\hline & Frambuesa & Rubus idaeus \\
\hline & Frutilla & Fragaria $\times$ ananassa \\
\hline
\end{tabular}


SABERES LOCALES EN HUERTAS DE MONTAÑA DEL SUR DE LOS ANDES: UN REFUGIO ... • 17

\begin{tabular}{|l|l|l|}
\hline \multicolumn{1}{|c|}{ Familia } & \multicolumn{1}{|c|}{ Nombre común } & Nombre científico \\
\hline \multirow{5}{*}{ Rosaceae } & Frutilla silvestre* & Fragaria chiloensis \\
\cline { 2 - 3 } & Guindo & Prunus cerasus \\
\cline { 2 - 3 } & Manzano & Malus domestica \\
\cline { 2 - 3 } & Membrillo & Cydonia oblonga \\
\cline { 2 - 3 } & Mosqueta** & Rosa eglanteria \\
\cline { 2 - 3 } & Murra/Mora** & Rubus ulmifolius \\
\cline { 2 - 3 } & Peral & Pyrus communis \\
\cline { 2 - 3 } & Ruda** & Ruta chalepensis \\
\hline Salicaceae & Mimbre** & Salix viminalis \\
\hline Santalaceae & Quinchamalí* & Quinchamalium chilense \\
\hline Scrophulariaceae & Matico** & Buddleja globosa \\
\hline \multirow{5}{*}{ Solanaceae } & Ají cacho de cabra, Pimentón & Capsicum annum \\
\cline { 2 - 3 } & Ají verde & Capsicum baccatum \\
\cline { 2 - 3 } & Natre** & Solanum ligustrinum \\
\cline { 2 - 3 } & Palqui* & Cestrum parqui \\
\cline { 2 - 3 } & Papa & Solanum tuberosum \\
\cline { 2 - 3 } & Tomate, Tomate cherry & Lycopersicum esculentum \\
\hline Urticaceae & Ortiga** & Artica dioica \\
\hline Verbenaceae & Cedrón & Drimys winteri \\
\hline Winteraceae & Canelo** & \\
\hline
\end{tabular}

* Especie de recolección.

** Especie que puede ser tanto recolectada como cultivada intencionalmente.

Fuente: Elaboración propia. 